Supporting Information

\title{
Highly Stable Gold(III) Complex with a Hydantoin Ligand in Alkaline Media
}

Kenichi Oyaizu, Yutaka Ohtani, Asako Shiozawa, Kenji Sugawara, Takashi, Saito, and Makoto Yuasa*

Institute of Colloid and Interface Science, Tokyo University of Science, Tokyo 162-8601, Japan, Department of Pure \& Applied Chemistry, Faculty of Science and Technology, Tokyo University of Science, Noda 278-8510, Japan, and Tanaka Kikinzoku Kogyo K. K., Hiratsuka 254-0076, Japan

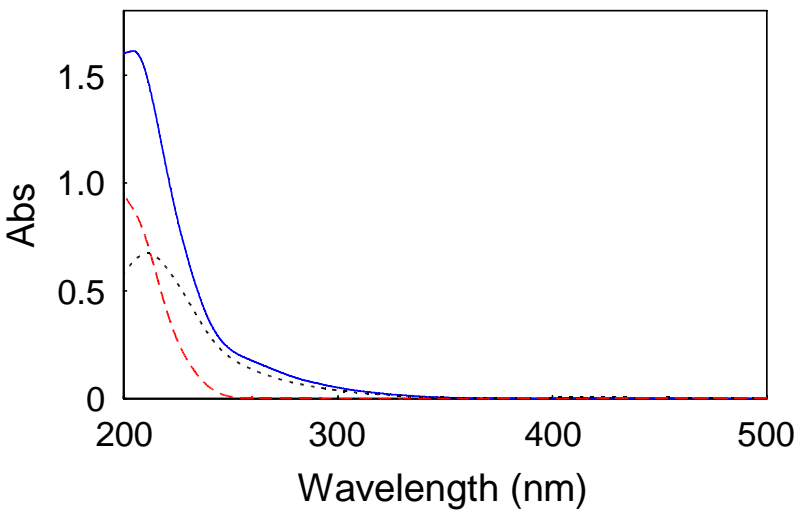

Figure S1. UV-vis. spectra for a solution prepared by dissolving Hdmh (0.24 mM) in the aqueous buffer at $\mathrm{pH} 8$ in the absence (red dashed curve) and presence (blue solid curve) of $\mathrm{HAuCl}_{4} \cdot 4 \mathrm{H}_{2} \mathrm{O}(0.04 \mathrm{mM})$. Black dotted curve corresponds to the spectrum for a solution prepared by dissolving $\mathrm{HAuCl}_{4} \cdot 4 \mathrm{H}_{2} \mathrm{O}(0.04 \mathrm{mM})$ in the same buffer. A shoulder near $265 \mathrm{~nm}$ corresponds to the absorption due to the $\left[\mathrm{Au}(\mathrm{dmh})_{4}\right]^{-}$complex. The optical path length was $1 \mathrm{~cm}$. 